\title{
Teoría de Popper y los riesgos en la ingeniería
}

\section{RESUMEN}

En el presente artículo se presenta las teorías del filósofo Karl Raimund Popper sobre el método hipotético deductivo, los problemas de la inducción y demarcación. Se presenta conceptos sobre ciencia, tecnología e ingeniería y las normas de estandarización. Se desarrolla la teoría de riesgos relacionado a los proyectos de ingeniería.

Palabras clave: epistemología, riesgos en ingeniería, ciencia

POPPER THEORY AND RISK IN ENGINEERING

\section{ABSTRACT}

This article is outlined the theories of the philosopher Karl Raimund Popper on hypothetical deductive method, problems of induction and demarcation. It is presented concepts of science, technology and engineering and standardization norm. It is developed the theory of risks related to engineering projects

Keywords: epistemology, risk in engineering, science

\section{INTRODUCCIÓN}

Karl Raimund Popper (1902-1994) vivió en un siglo que se caracterizó por las dos guerras mundiales y por un avance significativo del desarrollo científico. En los primeros años del siglo XX las teorías filosóficas de Emmanuel Kant (17241804), David Hume (1711-1779) y Auguste Comte (1788-1857) tenían una gran importancia, en los años 1922-1936 surge un movimiento científico y filosófico llamado el Círculo de Viena, que aglutinó a los filósofos más renombrados de Europa, se ocuparon principalmente de la lógica de la ciencia, elaborando un lenguaje común a todas las ciencias. Popper tuvo buenas relaciones con este círculo aunque con ciertas discrepancias.

Popper publica en 1945 su obra en filosofía social y política La Sociedad Abierta y sus Enemigos [6], en la que efectúa una defensa de la democracia. En 1957 publica su libro La Miseria del Historicismo, hace defensa de la libertad y la democracia liberal, a su juicio, por las concepciones autoritarias (marxistas y fascistas). En 1959 publica La Lógica de la Investigación Científica [5], propone la tesis sobre el modo cómo se acrecienta y desarrolla el conocimiento humano en general. En 1963 en su obra Conjeturas y Refutaciones [4] desarrolla temas sobre el desarrollo del conocimiento científico.

Popper, en su obra La Lógica de la Investigación Científica, afirma que la epistemología se ocupa del aumento del conocimiento científico, el cual resulta de la contrastación de las hipótesis o teorías, de modo que el problema central es de orden metodológico. Las dos cuestiones fundamentales de la teoría de Popper son:

- El problema de la inducción, que es la inferencia que se hace de enunciados particulares a enunciados universales, por más observaciones que se tengan, un día cualquiera las teorías pueden resultar falsas. La verdad de los enunciados particulares, de ninguna manera garantiza la verdad de los enunciados universales.

- El problema de la demarcación, que consiste en encontrar un criterio que distinga los enunciados empíricos de los metafísicos. El hombre de ciencia, ya sea teórico o experimental, propone enunciados, o sistemas de enunciados,

* Docente de la Facultad de Ingeniería Industrial, UNMSM. E-mail: orojasla@hotmail.com ** Docente de la Facultad Ingeniería Industrial, UNMSM. E-mail: danielmavila@yahoo.es

*** Docente de la Facultad Ingeniería de Sistemas, UNMSM. E-mail: cortez augusto@yahoo.fr 
y los contrasta paso a paso. Popper propone como criterio de demarcación la falsabilidad con base en la observación y la experiencia; estas permiten refutar o corroborar las hipótesis o las teorías, comprobar la hipótesis buscando hechos que puedan falsarla, si no se encuentran se considera provisionalmente verdadera hasta que se descubra un caso que falsee la hipótesis y pueda así ser descartada.

Ciencia e ingeniería tienen diferentes métodos y buscan diferentes objetivos, Popper señala que "el indeterminismo y el libre albedrío han pasado a ser parte de las ciencias físicas y biológicas". La ciencia busca una verdad universal, la ingeniería no se fija ni en la verdad ni en la universalidad, el fin del ingeniero es utilitario, mientras que la teleología del científico es cognitiva. Los ingenieros contribuyen a la sociedad a través de su habilidad para aplicar principios científicos básicos a problemas reales de una forma eficiente y efectiva. Se emplea el método científico para desarrollar modelos que expliquen fenómenos reales.

Las distintas normas estandarizadas que rigen en la ingeniería son desarrolladas en base a los acontecimientos, la experiencia y el desarrollo de los conocimientos. En la publicación de Solari [9] se menciona: en 1910 la cantidad de explosiones de calderas en Estados Unidos alcanzó la alarmante cifra de 1400 al año, los ingenieros estadounidenses establecieron normas de seguridad que minimizaron drásticamente el problema. American Society of Mechanical Engineers (ASME), elaboró por primera vez en 1914 el Boiler and Pressure Vessel Code (BPVC). Actualmente es empleado para el diseño, construcción, inspección, y ensayos de recipientes a presión. El Código ASME es la resultante de esos logros y fracasos, esta norma fue adoptada por Estados Unidos y muchos otros países, el código adquirió el carácter de American National Standard, y desde 1972 constituye un International Standard, en virtud de los programas internacionales de acreditación. Los códigos y normas son un reflejo de un acuerdo entre partes, productores, usuarios y técnicos, constituyen un mínimo que se debe cumplir, aunque el "estado del arte" está por encima del conocimiento reglamentado. Durante un juicio multimillonario por fallas durante la construcción de una plataforma petrolera off-shore en el Mar del Norte, un juez inglés falló en contra de los diseñadores a pesar de que estos habían cumplido exactamente con todos los requerimientos de los códigos y normas contractuales de la British Standard, se argumentó que el ingeniero debe conocer y cumplir con el "estado del arte", y era conocido entre los expertos de la época que algunos mecanismos de falla aún no estaban suficientemente considerados en las normas británicas aplicables, pero sí en normas empleadas en otros países como Japón.

Si bien el riesgo está presente en el trabajo del ingeniero, los deberes y obligaciones morales se tienen que cumplir. Desde antes de Cristo se consideran los daños a terceros; en el código de Hammurabi 1760 a. C. se establece "si un albañil ha edificado una casa para un señor, pero no ha dado solidez a la obra y la casa que construyó se ha desplomado y ha causado la muerte del propietario, ese albañil recibirá la muerte". En el código deontológico del Colegio de Ingenieros del Perú en su artículo $5^{\circ}$ se indica "Los ingenieros están al servicio de la sociedad. Por consiguiente tienen la obligación de contribuir al bienestar humano, dando importancia primordial a la seguridad y adecuada utilización de los recursos en el desempeño de sus tareas profesionales" [11].

Las condiciones en las que los individuos y organizaciones toman decisiones son reflejo de las fuerzas del entorno (sucesos y hechos), y generalmente se cuenta con limitada información que no se pueden controlar, pero que pueden influir a futuro en los resultados de sus decisiones, estas decisiones tienen asociadas un cierto grado de riesgo y se basan en la percepción y tolerancia de cada persona y organización, por lo que antes de decidir se hace un análisis que permita al interesado valorar la mayor cantidad posible de factores asociados. Los diferentes criterios de decisión en ambiente de riesgo se basan en estadísticos asociados a la distribución de probabilidad de los resultados, por lo que las organizaciones, desarrollan modelos estadísticos, con el objeto de pronosticar variables económicas, ambientales, políticas y sociales.

Un problema puede tener muchas posibles soluciones, y calcular los resultados esperados de todas ellas puede ser extremadamente lento y costoso, las situaciones de toma de decisiones se consideran dentro de una línea continua que va de la certeza, escenario altamente previsible, a la turbulencia escenario altamente imprevisible. Para minimizar o evitar las consecuencias catastróficas asociadas con accidentes, la ingeniería dispone de métodos técnicos y sociales que permiten la toma de decisiones capaces de minimizar las pérdidas. El empleo de iniciativas basadas en el riesgo ha sido incorporado a la ingeniería como herramienta para medir y evaluar los riesgos, hacer un ranking de criticidad de las instalaciones o componentes, decidir acciones de mitigación, aceptación y 
comunicación de los riesgos, gestionar los riesgos. La idea es decidir e implementar acciones preventivas antes que ocurra un accidente con consecuencias catastróficas.

Un sistema de gestión de riesgos es una aproximación a la gestión de sistemas industriales, basada en la identificación y control de aquellos eventos peligrosos, que tienen el potencial de causar cambios no deseados con consecuencias catastróficas. Su implementación permite operar las plantas industriales con seguridad, confiabilidad, disponibilidad y rentabilidad. Una adecuada gestión de riesgos requiere considerar los siguientes aspectos previamente analizados: identificar peligros/amenazas, integrar la información relevante en una base de datos, analizar y evaluar riesgos, mitigar los riesgos, aceptar los riesgos remanentes, y comunicar los riesgos.

Tanto la inducción como la demarcación tienen bastante relación con el trabajo del ingeniero, para el presente artículo se orientará al tema de los riesgos que se halla en forma implícita en los proyectos de ingeniería dado que el riesgo acompaña a todo cambio e implica elección e incertidumbre.

Popper, en su autobiografía [3] presenta una reflexión que los ingenieros constantemente tienen presente: "... porque fue mi maestro quien me enseñó no solamente cuan poco sabía, sino también que cualquiera que fuese el tipo de sabiduría a la que yo pudiese aspirar jamás, no podría consistir en otra cosa que en percatarme más plenamente de la infinitud de mi ignorancia".

Carlos Verdugo [10] resume los pensamientos importantes de Popper.

\section{LAS TEORÍAS DE POPPER}

Toda observación se hace desde un punto de vista y que por ello, toda observación tiene matices derivados de nuestra forma de pensar. El mundo se presenta en el contexto de las teorías que se asume. La observación está "teóricamente influida". Aunque haya muchas instancias que confirmen una teoría, solo se necesita una contra observación, que la falsee. Como ejemplo para explicar la confirmación de una teoría en diferentes escritos se presenta la metáfora del Cuadro 1.

La generalización sería imposible de corroborar, pues se tendría que buscar a todos los cisnes que existen; pero si se propone falsar la generalización se podría hacer lo contrario, se buscaría un cisne de cualquier color que no fuera blanco, si se encontrara, se habría falseado o refutado la generalización. Ejemplos para explicar el concepto de falsabilidad:

1. Todos los cisnes son blancos.

2. Los metales se dilatan al calentarlos.

3. La masa de un protón es de 1,6726 × $10^{-27} \mathrm{~kg}$.

4. Es probable que suba la cotización de las acciones comunes de la empresa Minas Buenaventura.

5. En grupo de isometría de un círculo, la totalidad de los puntos del círculo euclídeo equidistan del centro.

La primera afirmación es falsable y además falsa debido a que es posible encontrar un cisne de un color diferente del blanco. El segundo enunciado también se puede falsar, únicamente es necesario

Cuadro 1. Teoría de Popper
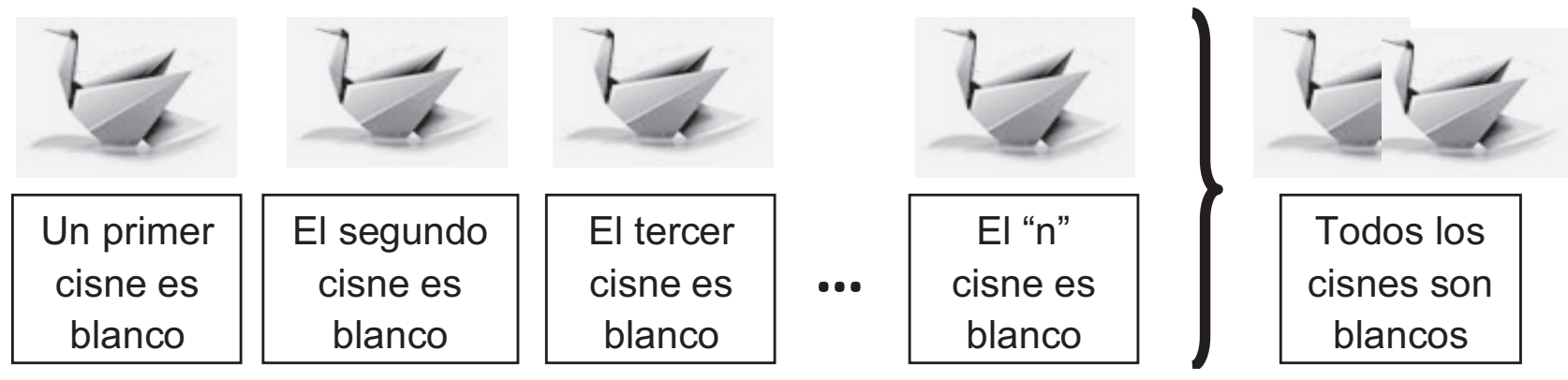

Fuente: http://www.serguel.com/post/los-3-mundos-de-karl-popper-y-un-globo-rojo\#more-2422 
encontrar un metal que no se dilate al calentarlo. El tercer caso también es falsable, dado que se demostraría como falso si se encuentra un protón que tenga una masa diferente. Según el falsacionismo, estos tres casos son científicos. El primero no se utilizará porque se ha demostrado falso, pero los otros pueden formar parte del cuerpo de una teoría científica válida hoy en día. La afirmación número cuatro no es falsable. Si la cotización de las acciones comunes de Minas Buenaventura sube, la afirmación es cierta, pero si baja o se mantiene igual también lo es. Es imposible encontrar un enunciado simple que la contradiga. La última afirmación no es falsable considerando la definición de círculo porque si alguno de los puntos no estuviese a la misma distancia del centro que el resto de puntos, entonces ya no sería un círculo. Por lo tanto los dos últimos enunciados no son científicos.

Los tres primeros ejemplos han de considerarse científicos porque aportan información, nos dicen cosas sobre el mundo, los dos últimos no dicen nada. La afirmación "Todos los planetas se mueven alrededor del sol siguiendo órbitas elípticas" es científica, porque indica que los planetas giran alrededor del sol y no en otra parte, y además que lo hacen siguiendo unas órbitas en forma de elipse y no cuadradas o triangulares. Además es falsable, porque podría existir un planeta que no siguiese este tipo de órbitas o girase alrededor de otra cosa; este enunciado debería ser rechazado, porque existen planetas que no orbitan alrededor del sol, sino alrededor de otras estrellas. Aún así tiene el carácter de científico, pero no se utilizará porque ha sido falsado.

Popper establece que las teorías se construyen como conjeturas o suposiciones especulativas y provisionales que el intelecto crea en un intento de solucionar problemas y de proporcionar una explicación adecuada del comportamiento de algunos aspectos del mundo o el universo, las que deberán ser comprobadas en forma rigurosa por la observación y/o la experimentación; las teorías que no superen las pruebas deben ser eliminadas y reemplazadas por otras. Todo el conocimiento humano y las ciencias son conjeturas, somos falibles y la ciencia también lo es, no hay certeza en el conocimiento humano. Ninguna teoría puede ser considerada alguna vez como establecida o verificada en forma concluyente y definitiva. El método de conjeturas y refutaciones, es el instrumento principal del crecimiento científico y puede ser concebido en términos más generales como la actitud crítica o racional. La diferencia fundamental entre el conocimiento común y aquel de carácter científico consiste en que este último es consciente y planificador en detectar nuestros errores con el fin de eliminarlos.

La ciencia progresa cuando se demuestra que una teoría está equivocada y se introduce una nueva teoría que explica mejor los fenómenos, es de suponer que en el futuro se propondrán nuevas falsaciones. El conocimiento científico no avanza confirmando nuevas leyes, sino descartando leyes que contradicen la experiencia. Cuando las hipótesis superan la falsación y han sido contrastadas se convierten en leyes científicas que tienen el carácter de provisionalidad. Popper exige que los enunciados sean potencialmente falsables a partir de la experiencia empírica. Aquellos enunciados que no tienen la posibilidad de ser falsables no pertenecen a la ciencia y por tanto no son objetivos.

En el Cuadro 2, se presenta un esquema mostrando cómo se llega al conocimiento científico partiendo del falsacionismo.

El criterio de demarcación es la capacidad de una proposición de ser refutada o falsada. Solo se admitirán como proposiciones científicas aquellas para las que sea conceptualmente posible un experimento o una observación que las contradiga. No todo enunciado es científico solo lo será aquel que potencialmente sea empíricamente refutado por los hechos. El criterio de demarcación entre ciencia y no ciencia, según Popper es que "teorías científicas son aquellas que están potencialmente abiertas al falseamiento", por ejemplo, dentro de la ciencia quedan la teoría de la relatividad y la mecánica cuántica, y fuera de ella, el marxismo o el psicoanálisis. Popper concibió el criterio de demarcación interesado en encontrar una frontera entre lo científico y lo metafísico.

Como ejemplos para diferenciar entre ciencia y metafísica se plantea:

A) Ciencia: la ley de la gravitación universal, la fuerza ejercida entre dos cuerpos de masas $m_{1}$ y $m_{2}$ separados una distancia $r$ es proporcional al producto de sus masas e inversamente proporcional al cuadrado de la distancia, es decir:

$$
F=G \frac{m_{1} m_{2}}{r^{2}}
$$

Donde: $F$ es el módulo de la fuerza ejercida entre ambos cuerpos, y su dirección se encuentra en el eje que une ambos cuerpos y $G$ es la constante de la gravitación universal.

B) Metafísica: pensaré en ti después de mi muerte. 
Cuadro 2. El conocimiento científico a partir del falsacionismo

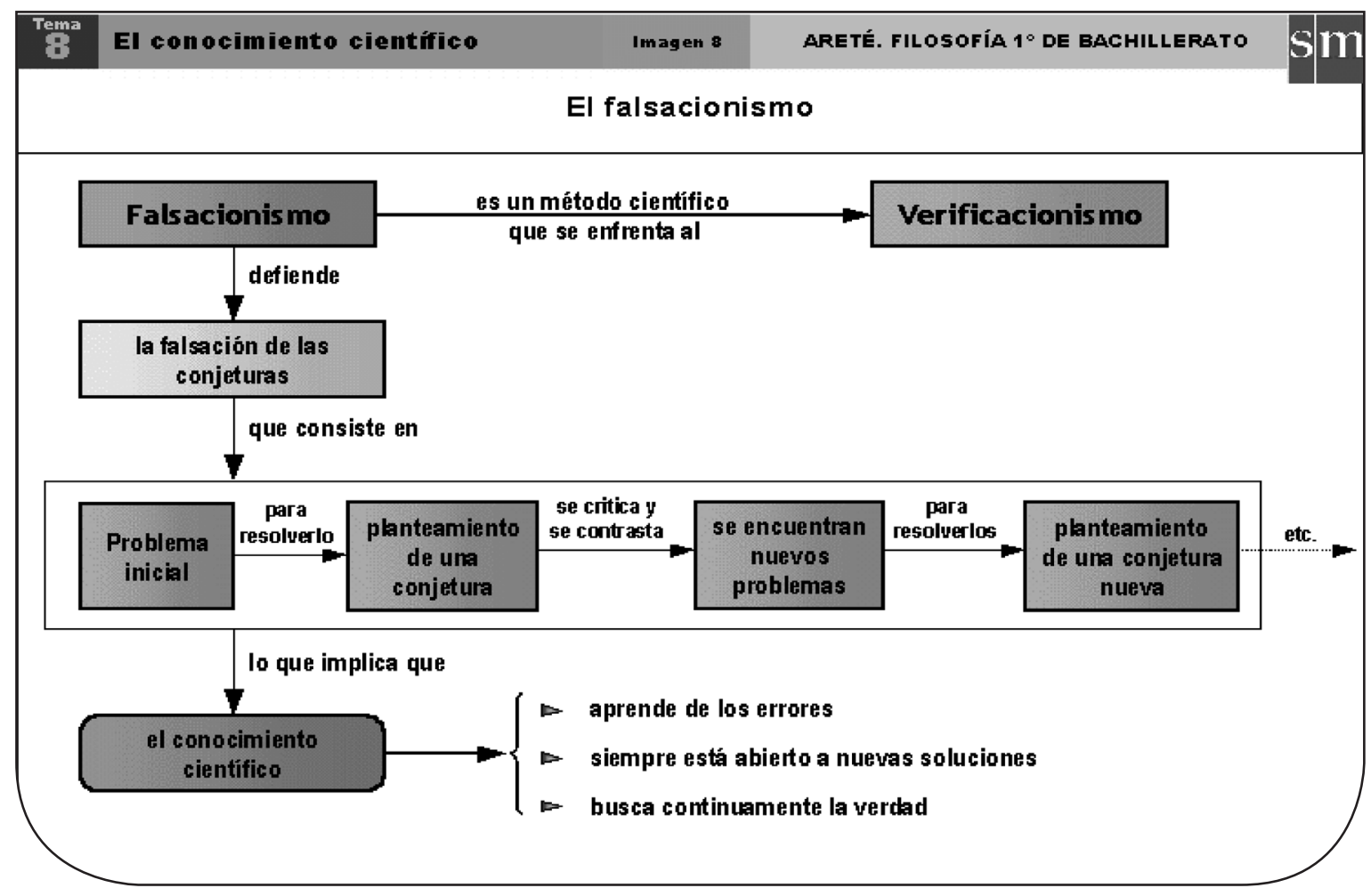

Fuente: http://www.ite.educacion.es/w3/recursos/bachillerato/filosofia/aula_filosofia/filosofia1/apuntes1/ApuntesCiencia.htm

En el método hipotético deductivo el científico se enfrenta o selecciona un problema interesante o importante. A continuación propone una solución tentativa o conjetural en la forma de una hipótesis o de una teoría científica. El próximo paso consiste en criticar la hipótesis lo mejor que se pueda, esto es, se intenta refutarla a través de las contrastaciones o controles más severos que se puedan diseñar. Si la hipótesis o teoría resiste y sobrevive estos serios y rigurosos intentos de refutación o falsación, ella es considerada como exitosa y aceptada provisoriamente (Cuadro 3).

\section{LOS TRES MUNDOS SURREALISTAS DE POPPER}

La epistemología se ha fundamentado en su base teórica en Popper, quien trata de indicar que todo aquello que existe habita en tres mundos que son los siguientes:

a) Mundo 1: es el lugar donde existen los sucesos y fenómenos (se utiliza la razón lógica sin ninguna prueba científica). b) Mundo 2: se desarrollan las teorías subjetivas (utilizar la razón psicológica la cual nos da a entender de diferente manera las cosas, para cada uno de nosotros).

c) Mundo 3: rechaza las teorías falsadas y acepta las que no han sido falsadas aún. En este mundo se da la falsación, para Popper la esencia de la ciencia. En la base de los conocimientos científicos, se encuentra la cultura, el arte y la ciencia.

Para poder entender estos conceptos es común presentar el ejemplo del globo rojo:

- Mundo 1: un globo rojo en un día soleado crece de tamaño al pasar cierto tiempo.

- Mundo 2: el globo crece 0,25 cada dos horas, por lo que se puede formular $Y=0,125 X$. Cuadro 4 (a).

- Mundo 3: después de las 6 horas el globo decrece. Cuadros $\mathrm{N}^{\circ} 4$ (b) y N4 (c).

Popper señala que la experiencia permite concebir teorías, mismas que después se someten a experimentos con el propósito de corroborarlas o refutarlas. 
Cuadro 3. Método hipotético deductivo

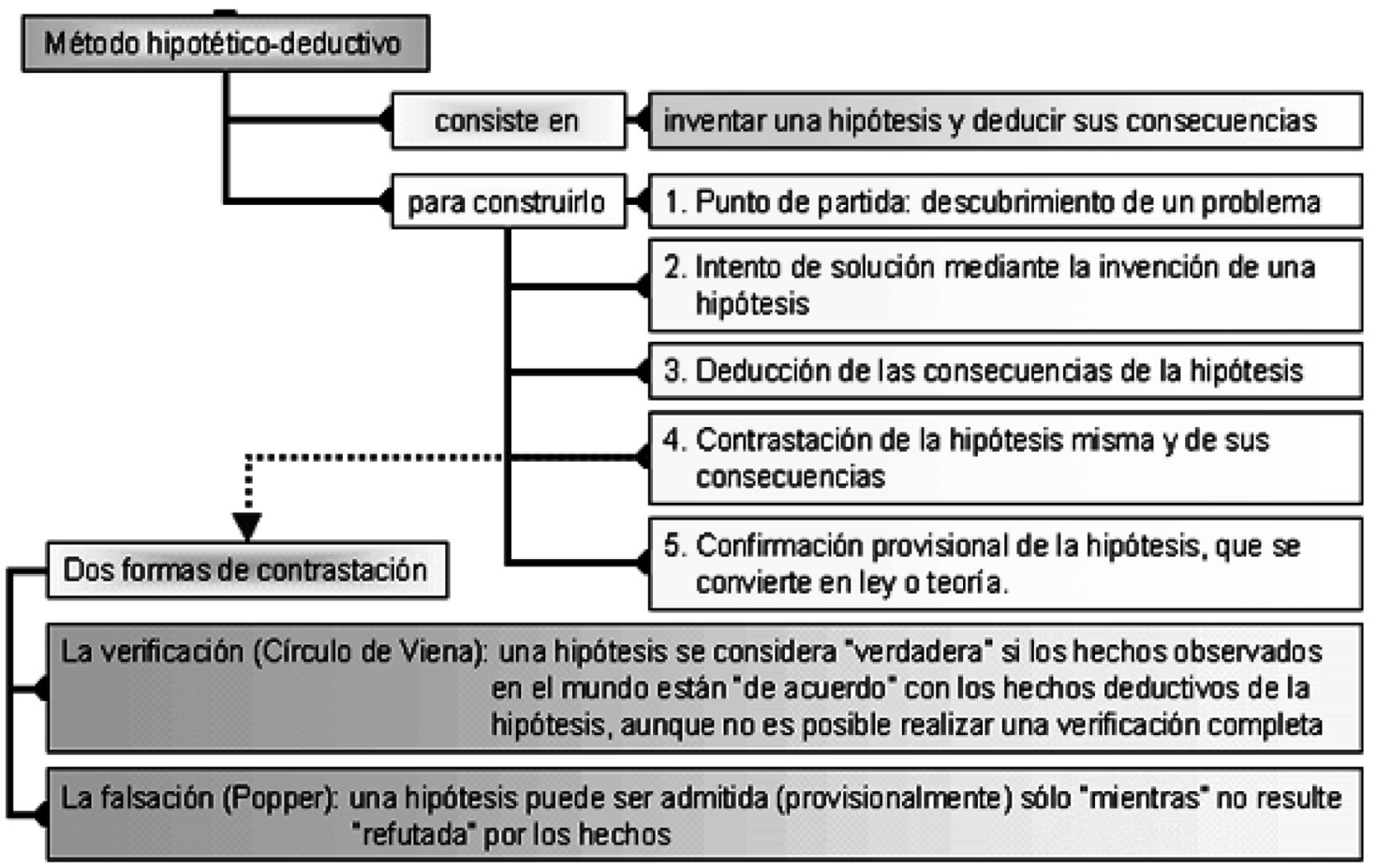

Fuente: http://www.ite.educacion.es/w3/recursos/bachillerato/filosofia/aula_filosofia/filosofia1/apuntes1/ApuntesCiencia.htm

\section{Cuadro 4. Representación del globo rojo}

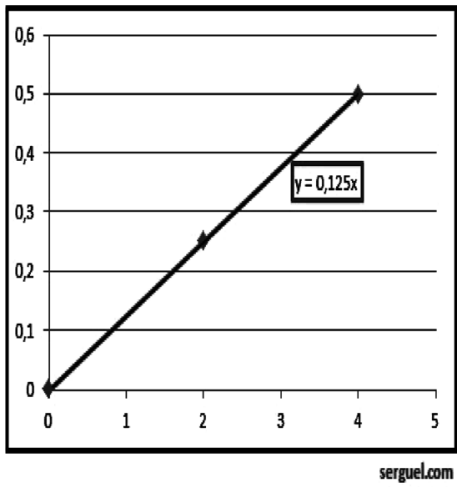

(a)

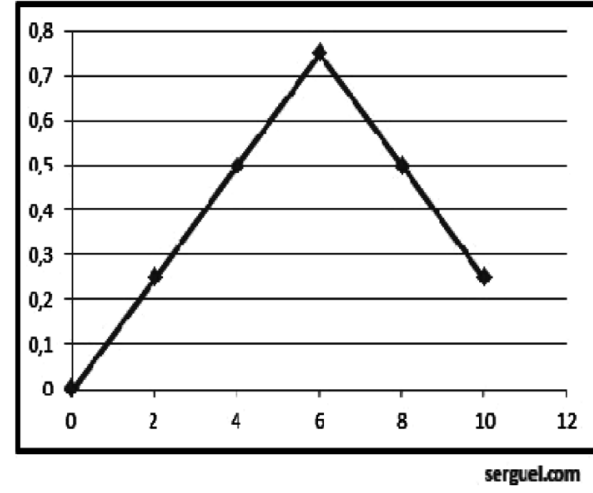

(b)

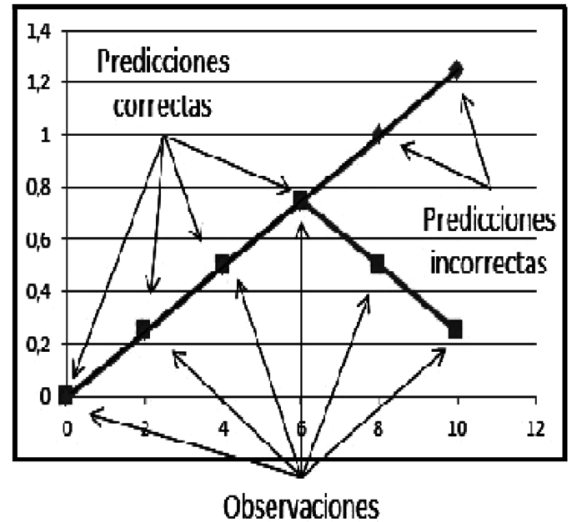

(c)

Fuente: http://www.serguel.com/post/los-3-mundos-de-karl-popper-y-un-globo-rojo\#more-2422 
Según Antonio Núñez [1], las diferencias entre ciencia, tecnología e ingeniería se muestra a continuación:

- La ciencia estudia la naturaleza, sus objetos y fenómenos y sus procesos y cambios. $Y$ la motivación es la curiosidad, el querer entender, busca el qué y el porqué, qué pasa, qué hay, por qué.

- La ciencia aplicada ejecuta la ciencia experimental en los ámbitos de la utilidad, a la solución o remedio de algún problema, o a dar solución a las necesidades que demanda la tecnología.

- La tecnología es la aplicación del conocimiento científico y de los procedimientos técnicos a la realización de tareas prácticas por medio de sistemas organizados que comprenden personas y organizaciones, seres vivos $y$ máquinas. La tecnología es la ciencia aplicada, busca el cómo y el para qué.

- La ingeniería estudia el objeto artificial y sus procesos como la tecnología, pero está centrada en la solución de problemas de interés para el hombre, y considera su construcción y los recursos, la economía, la realidad de que existen recursos escasos para la construcción de esos objetos útiles, y por eso considera eficiencia en costes y rendimientos, seguridad para la vida humana, la aceptación del público y la manufacturabilidad de sus obras. En todo ello busca cómo hacerlo, al considerar los procesos complejos surge también el observar y ver qué pasa, qué hay, el porqué y para qué del proceso constructivo. Los ingenieros producen mediante el proceso creativo llamado diseño de un producto que usualmente es un dispositivo físico, una estructura o un proceso.

- La innovación se centra en renovar y hacer cambios para mejorar algo existente. La novedad es consecuencia del cambio y de la mejora. La mejora no requiere necesariamente introducir un nuevo producto. Se innova en organización, en decisiones, en procesos, en industrialización y en productos.

Según la Oficina de Acreditación para Ingeniería y Tecnología (ABET, por sus siglas en inglés) se define a la ingeniería como la profesión en la cual los conocimientos de las matemáticas y las ciencias naturales alcanzadas mediante el estudio, la experiencia y la práctica son aplicadas con juicio para desarrollar formas de utilizar económicamente los materiales y fuerzas de la naturaleza en beneficio de la humanidad.
Según la Federación Europea de Asociaciones Nacionales de Ingenieros (FEANI) define a la ingeniería como: "La actividad profesional que consiste en la aplicación creativa de los conocimientos científico-técnicos a la invención, desarrollo y producción de bienes y servicios, transformando y organizando los recursos naturales para resolver las necesidades del hombre, haciéndolo de una manera óptima, tanto económica como socialmente".

En el campo de la ingeniería existe un conjunto de normas que rigen su accionar, entre las más importantes se tiene:

- Reglas de diseño probadas, normas técnicas nacionales e internacionales, donde los factores de seguridad cubren errores de diseño.

- No se habla de "ciencias de ingeniería".

- En el diseño de plantas y procesos se debe aprovechar la ley de la gravedad.

- Se debe buscar la mejor solución a un problema planteado considerando un conjunto de limitantes, siempre hay solución a un problema, así sea aproximada.

- Los problemas planteados generalmente son abiertos, admiten múltiples soluciones, y pueden ser sistemas complejos, partes interconectadas que contiene información no visible.

- Algunos problemas según sus restricciones admiten una solución única (por métodos matemáticos).

- El conocimiento del estado del arte, know-how, training, licencias de explotación, permisos, saber qué es lo que se sabe y quién lo sabe, está asociado a la mejor práctica en ingeniería.

- Uso de paradigmas y heurísmos ingenieriles (grandes modelos conceptuales).

- Uso de modelos, simulaciones que optimizan la tecnología.

- Tener en cuenta a los stakeholders del proyecto.

- Los errores sirven para analizar y mejorar las reglas de diseño.

En el Cuadro 5, se presenta una comparación entre el método científico y el método ingenieril.

Cuando al ingeniero se le presenta situaciones nuevas especiales deberá seguir pasos más cercanos al método científico, entonces podría tomar la secuencia mostrada en el Cuadro 6. 
Cuadro 5. Comparación entre el método científico y el método ingenieril
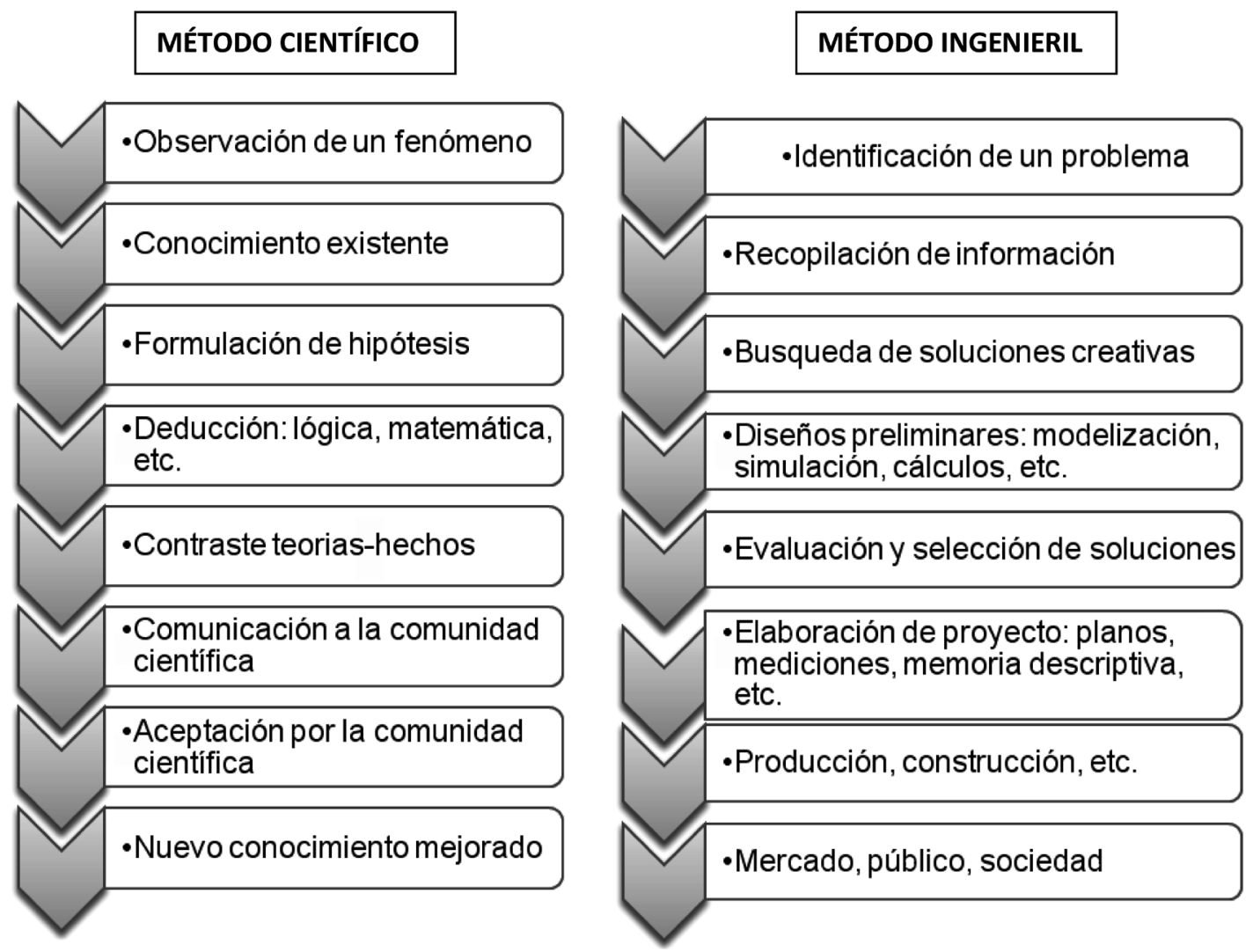

Fuente: V. Ortega. Ingeniería y Civilización [2].

Cuadro 6. Método ingenieril

-Formular el problema

- Decidir sobre las conjeturas

- Derivar consecuencias lógicas de las conjeturas

- Definir técnicas para el estudio de las conjeturas

- Cuestionar las técnicas para determinar su relevancia y fidelidad

- Contrastar las conjeturas aplicando las técnicas y evaluar sus resultados

- Estimar la pretensión de la verdad

- Determinar los dominios en los cuales son válidas las conjeturas y las técnicas

- Formular nuevos problemas originados por la investigación

Fuente: V. Ortega. Ingeniería y Civilización [2]. 


\section{EL RIESGO EN LA INGENIERÍA}

El riesgo, es la posibilidad de que suceda o no un daño, está relacionado con el peligro y la vulnerabilidad, no hay vulnerabilidad sin peligro. Según Popper la ciencia debe tomar riesgos. La ingeniería mide el riesgo como el producto entre la probabilidad, o frecuencia de ocurrencia, y las consecuencias o severidad de un peligro, limitándose su alcance a un ambiente específico y durante un periodo de tiempo determinado.

Las estrategias frente al riesgo pueden ser reactivas o proactivas y las categorías de los riesgos pueden ser relacionados con: tamaño del producto, impacto en la organización, tipo de cliente, definición del proceso de producción, entorno de desarrollo, tecnología, experiencia y tamaño del equipo, etc.

En condiciones de certidumbre, el ingeniero puede al menos prever (si no es que controlar) los hechos y sus resultados. Esta condición significa el debido conocimiento y clara definición tanto del problema como de las soluciones alternativas. El monto, tipo, calidad y confiabilidad de la información disponible influyen en el nivel de riesgo y en el hecho de si el responsable de tomar la decisión puede hacer uso de la probabilidad objetiva, posibilidad de que ocurra un resultado específico con base en hechos consumados, o subjetiva, juicios y opiniones personales de que ocurra un resultado específico, en la estimación del resultado.

En la toma de decisiones que pueden causar la incertidumbre y el riesgo, existen fenómenos no atribuibles directamente al que toma las decisiones, como por ejemplo:

- Inexistencia de datos históricos directamente relacionados con las alternativas que se estudian.

- Sesgos en el cálculo de datos o de eventos posibles.

- Análisis e interpretación erróneos de la información disponible, sesgo en los cálculos, baja confiabilidad de los datos estadísticos, etc.

- Cambios en la economía, en la política de países que en forma directa o indirecta afectan el entorno económico.

- Obsolescencia debido al cambio tecnológico rápido.

- Catástrofes naturales o comportamiento del clima.

- Introducción de nuevos insumos y/o productos.
Para dar una efectiva respuesta a los desafíos de la población, la ingeniería como emergente de la cultura, debe configurarse considerando los miedos, las incertidumbres y las tendencias destructivas de la cultura que la emplea.

Popper introdujo una teoría objetivista: la teoría de la probabilidad como propensión. Esta teoría incluye las posibilidades con peso. Por ejemplo si un dado está cargado, existe una circunstancia física, el campo de propensiones, que ejerce su influencia en cada tirada en particular.

El primer paso del análisis es responder: ¿Qué puede salir mal? La respuesta lleva a definir diversos escenarios de riesgo. Algunas de las técnicas empleadas se basan en equipos de expertos, para que opinen en forma planificada, y en el análisis de los antecedentes técnicos disponibles. Estas técnicas simples intentan ser de sentido común sistematizado. Un problema, solo parcialmente resuelto, reside en la consideración de los escenarios impensables o improbables, es decir aquellos aspectos que, por desconocimiento o porque nunca fueron observados, quedan fuera del análisis.

Cuando la instalación o artefacto tiene un carácter único, en cuanto a que no hay antecedentes de haber sido construido anteriormente, la simulación computacional cobra una gran importancia, pero introduce incertidumbres, en algunos casos insalvables, por la falta de datos o historial de fallas genéricos. Tal como es el caso de las actualmente llamadas "megaconstrucciones".

El estado del arte de la ingeniería se basa en el método de prueba y error, iluminado por el método científico. Siempre que hay un nuevo desarrollo tecnológico, hay riesgos. El riesgo cero no existe. Cuando más experiencia histórica esté disponible, mayor será la capacidad de la ingeniería para minimizar los riesgos. Cuando se materializa un nuevo proyecto, para el que no hay antecedentes, los riesgos inicialmente serán altos, a medida que se aumente el conocimiento el riesgo disminuye, debido a mayor experiencia, resultados de ensayos en operación, accidentes, etc.

Las categorías generales de riesgos que generalmente se evalúan son: financieros, operacionales, interrupción del negocio, propiedad, desastres naturales, seguridad, salud ocupacional, público, ambiente, tecnológicos, obsolescencia, recursos humanos, dependencia de personas claves, propiedad intelectual, reputación, imagen pública, fraude, peligro moral, etc. 
Para cada escenario de riesgo definido, se deben contestar dos preguntas: ¿Cuál es su frecuencia o probabilidad de ocurrencia? y ¿Cuáles son sus consecuencias si se materializa el accidente? Con las respuestas se puede calcular el riesgo de cada escenario, como el producto entre la probabilidad y las consecuencias. Las consecuencias pueden medirse en términos económicos, de pérdidas de vidas humanas, área dañada, etc. La ingeniería dispone de métodos bien definidos y las herramientas matemáticas suficientes para calcular estas pérdidas con un buen grado de aproximación. Los modelos computacionales para calcular las consecuencias han evolucionado notablemente en los últimos años.

Con el transcurso del tiempo se puede producir un deterioro normal o acelerado. Por ejemplo, durante el diseño de una de las primeras plataformas petroleras off-shore del Mar del Norte, los ingenieros consideraron como peor escenario la "tormenta más grande del siglo". Refirió uno de los diseñadores que en el primer año de operación la estructura soportó, con serias dificultades, cinco "tormentas más grandes del siglo".

ASME emplea conjuntamente dos clases de probabilidades: objetivas y subjetivas. Las probabilidades que se derivan de los datos históricos de fallas corresponden a frecuencias objetivas, por ejemplo para un conjunto de 1000 tanques de almacenamiento de combustible, en los que se detectaron siete pequeñas pérdidas por el fondo en un año, corresponde una frecuencia anual de pérdidas de $7 \times 10^{-3}$. Sin embargo, aunque los datos sean precisos, es difícil contemplar, en la práctica que algunos componentes fallados hayan sido reparados y retornados al servicio o reemplazados. Las probabilidades subjetivas surgen de entrevistar en forma pautada a un experto acerca de cuándo puede ocurrir una falla. El conocimiento de los expertos, logrado tras largos años de estudio y experiencia, debe ser "extraído" empleando técnicas capaces de evitar su "contaminación" con las opiniones personales.

El Teorema de Bayes, o la denominada transformación bayesiana, es la herramienta generalmente empleada para combinar las diversas probabilidades. El enfoque bayesiano parte de una distribución de probabilidades a priori en la que el analista tiene un cierto grado de confianza antes de obtener datos objetivos, luego mediciones objetivas de la realidad (ensayos) modifican el grado de convicción inicial.

Popper criticó el empleo del enfoque bayesiano por considerar las probabilidades subjetivas y propuso reemplazar la teoría de probabilidades clásica por una teoría objetiva de probabilidades "pasadas",que denominó teoría de la probabilidad como propensión. Las propensiones o tendencias no son meras posibilidades, sino realidades físicas. Los actuales métodos de inspección basados en riesgo siguen algunos de los lineamientos de la teoría de las propensiones, combinándolos con frecuencias de ocurrencias históricas. En general, no se encuentran en los textos de ingeniería cuestionamientos, en el sentido de Popper al empleo de las probabilidades subjetivas, siendo su empleo una tendencia creciente en el contexto de API (American Petroleum Institute) y ASME.

El riesgo "técnico", tan objetivo como ha sido posible calcularlo, no es el riesgo "real", sino que es una medida de la percepción del riesgo. Cuanta más información se obtenga (resultados de ensayos, observaciones, etc.), se podrá modificar el riesgo percibido acercando al riesgo real que se desconoce.

Habiendo analizado los riesgos se debe compararlos con un valor de riesgo admisible para estar en condiciones de tomar decisiones. Un concepto muy empleado es el de riesgo As Low As Reasonably Practical (ALARP), que considera que el riesgo solo puede ser minimizado a un cierto nivel bajo la tecnología actual y a un costo razonable. Los riesgos son "aceptables" solo si se han tomado todas las medidas prácticas razonables para reducirlos, este nivel de riesgo está determinado por la ingeniería, como se observa en el Cuadro 7.

\section{Cuadro 7. Reducción del riesgo ALARP}

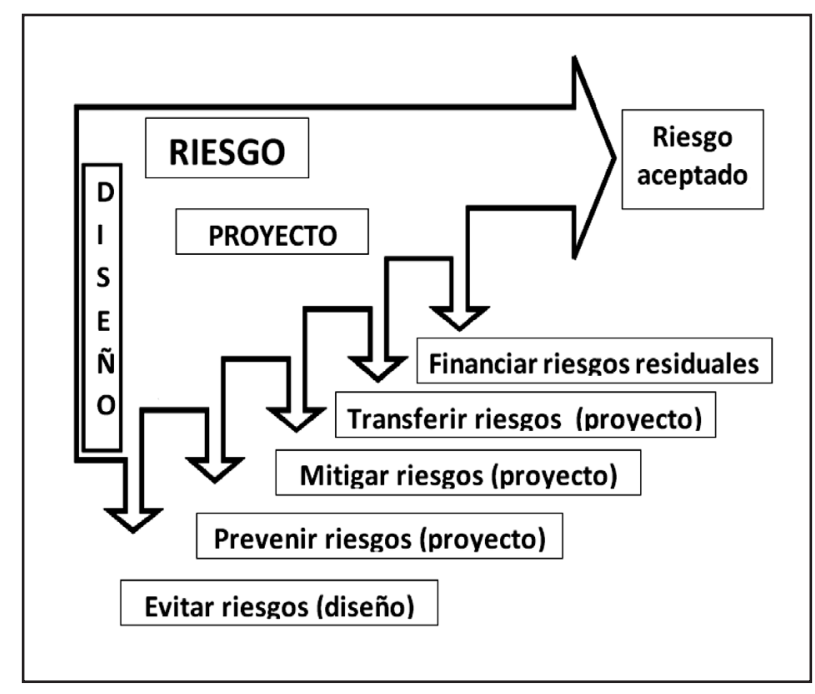

Fuente: Elaboración propia. 
El riesgo admisible ya no es solo un concepto técnico o experto, sino que es un concepto que involucra también la opinión de la sociedad. El nivel socialmente admisible debe ser determinado tanto por la tecnología como por el nivel de alarma de la sociedad, la conciencia ecológica de la población, un mismo riesgo es percibido de distintas maneras según difiera la perspectiva de los diversos países y culturas.

El diseño puede considerarse un proceso iterativo para concebir una idea con la intención de materializarla en un producto, que debe cumplir las funciones requeridas durante un periodo de tiempo previsto. Un buen diseño debe prevenir la ocurrencia de fallas durante el ciclo de vida del producto, satisfaciendo los requerimientos de seguridad, protección ambiental, y cumplimiento de las leyes y regulaciones. Los análisis de riesgo permiten identificar y corregir los posibles escenarios de falla durante la etapa de diseño. Esta metodología de trabajo se denomina Diseño Basado en Riesgo.

Las condiciones en las que se toman las decisiones pueden clasificarse en términos generales como certeza o certidumbre, incertidumbre y riesgo, en el Cuadro 8 se puede apreciar las probabilidades de ocurrencia versus los posibles resultados.

Certeza: se conoce la consecuencia de los resultados, información exacta, medible y confiable y sus probabilidades de ocurrencia de cada una de las alternativas de decisión, por lo que se pueden aplicar modelos computacionales de simulación.
Riesgo: hay varias posibles consecuencias para cada curso de acción y el tomador de decisiones no conoce por completo los resultados potenciales por lo que se asigna probabilidades de ocurrencia para obtener resultados deseados dentro de un margen deseable.

Bajo incertidumbre: hay varias posibles consecuencias para cada curso de acción, el tomador de decisiones no conoce la probabilidad de ocurrencia de las variables, no se pueden evaluar las interacciones de las diferentes variables. A medida que aumenta la incertidumbre, también aumenta la posibilidad de fracaso.

\section{CONCLUSIONES}

El racionalismo crítico de Popper está ligado al intento de construir un enfoque puramente deductivo de la ciencia, a su visión de la evaluación de las teorías y del aumento del conocimiento y al criterio de falsación y demarcación

Los problemas de la ingeniería tiene una dimensión técnica y otra social, los temas sociales han adquirido connotación mundial por lo que las soluciones dadas por los ingenieros deben ser parte de la solución y no parte del problema social.

La ingeniería puede controlar sus creaciones y minimizar la vulnerabilidad humana ante los desastres y las violencias ya que dispone de metodologías matemáticas y filosóficas, capaces de

Cuadro 8. Probabilidad de predecir resultados en la toma de decisiones

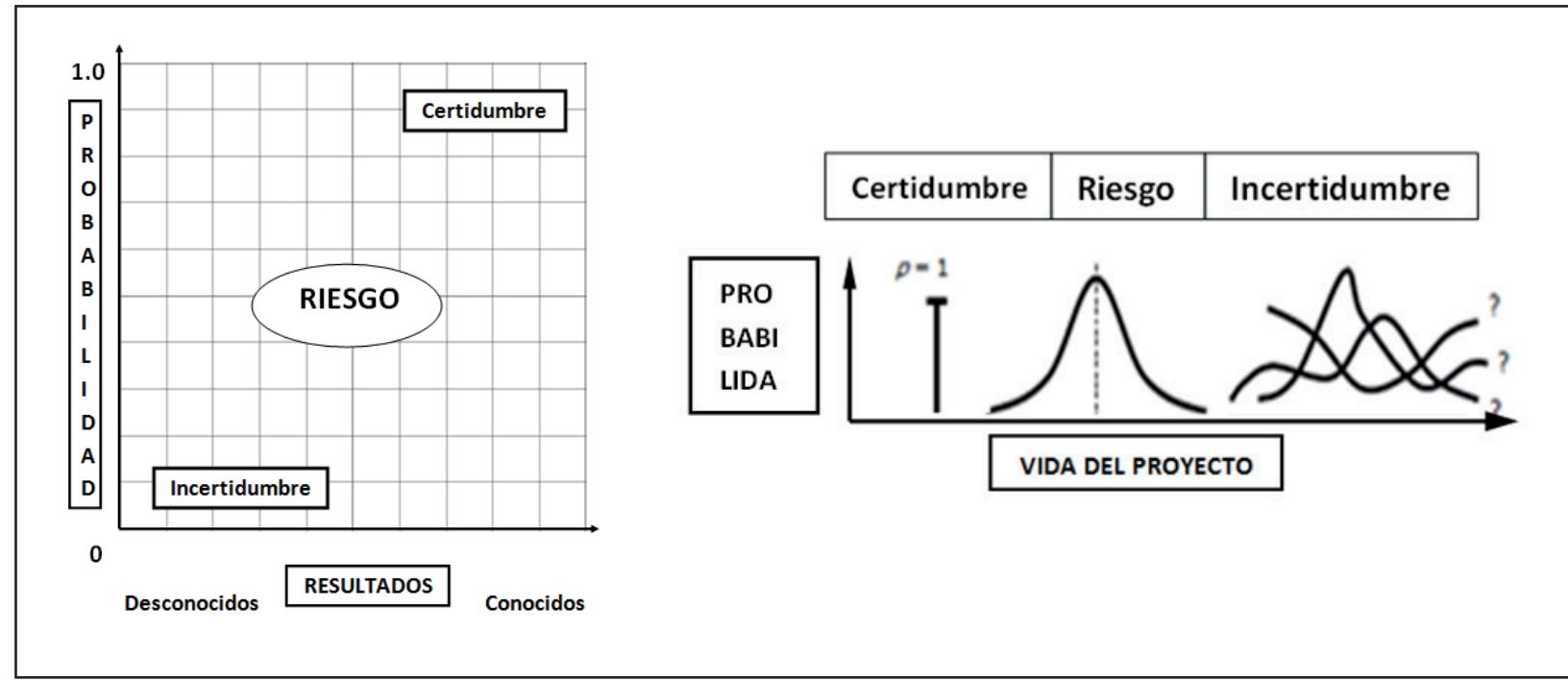

Fuente: Riggs, J. et al. [8] 
analizar y evaluar los riesgos "técnicos". Su empleo está bien establecido en los estándares industriales. Con más progreso científico y tecnológico el riesgo de desastres tenderá a disminuir.

Las empresas deben contar con una plana gerencial capacitada en la toma de decisiones bajo condiciones de certeza, incertidumbre y riesgo, pues su importancia es capital para la eficacia y eficiencia de la correcta toma de decisiones

Los ingenieros deben ampliar su visión al campo de la sociología y psicología para poder mejorar el empleo de las herramientas de confiabilidad humana, y además poder mejorar el diálogo con la sociedad con la que debemos compartir las decisiones sobre el riesgo, de manera de responder mejor a la pregunta: ¿cuál es un nivel de riesgo aceptable?

En el camino del desarrollo de la ingeniería se debe avanzar aplicando ciencia, creatividad, tecnología, percepción de la realidad y ética, de manera que los riesgos de un proyecto sean mínimos.

\section{REFERENCIAS BIBLIOGRÁFICAS}

[1] Núñez, A. www.iuma.ulpgc.es/ nunez/.../ Tema1Metodos/GuionTema1Metodos.ppt Visitado el 12 de mayo 2013.

[2] Ortega, V. Ingeniería y civilización. http:// es.scribd.com/doc/54079477/Ingenieria-yCivilizacion-TODAS-31-03-08. Visitado el 12 de mayo 2013.

[3] Popper, K. 1985. Búsqueda sin término. Una autobiografía intelectual. Editorial Tecnos. $288 \mathrm{pp}$.
[4] Popper, K. 1994. Conjeturas y Refutaciones: El Desarrollo del Conocimiento Científico. 2. ${ }^{a}$ ed. Editorial Paidós. 451 pp.

[5] Popper, K. 2002. La lógica de la investigación científica. 2. ${ }^{\text {a }}$ ed. Routledge. 513 pp.

[6] Popper, K. 2010. La Sociedad Abierta y sus Enemigos. Editorial Paidós, 704 pp.

[7] Redman, D. La teoría de la ciencia de Karl Popper: auge y caída de la ingeniería social.

[8] Riggs, J. et al. 2006. Ingeniería Económica. Editorial Alfaomega. 638 pp.

[9] Solari, M. La ingeniería en la sociedad del riesgo. Conferencia del Dr. Ing. Mario J. A. Solari en la Academia Nacional de Ciencias de Buenos Aires, en la sesión pública del 3 de noviembre de 2010. http://ciencias.org.ar/user/ FILE/Solari.pdf. Visitado el 15 de mayo 2013.

[10] Verdugo, C. Documento: Selección de escritos socio políticos de Karl Popper. http://www. tomasmoro.net/archivos/jpgaleano/docs/ideaspoliticas/Textos\%20de\%20Karl\%20Popper.pdf Visitado el 15 de mayo 2013.

[11] http://www.cip.org.pe/Cvista/publicaciones/ documentos/CODIGO_DEONTOLOGICO2012.pdf. Visitado 17 de mayo 2013.

[12] Los 3 mundos de Karl Popper y un globo rojo http://www.serguel.com/post/los-3-mundosde-karl-popper-y-un-globo-rojo\#more-2422. Visitado 15 de octubre 2012

[13] Tema 4 el saber científico archivo ppt http:// www.ite.educacion.es/w3/recursos/bachillerato/ filosofia/aula_filosofia/filosofia1/apuntes1/ ApuntesCienciā.htm. Visitado 10 de octubre 2012 